\title{
TRIM29 promotes progression of thyroid carcinoma via activating P13K/AKT signaling pathway
}

\author{
JINKAI XU, ZONGYU LI, QINGHUA SU, JUN ZHAO and JIANCANG MA \\ Department of General Surgery, The Second Affiliated Hospital of Xi'an Jiaotong University, \\ Xi'an, Shaanxi 710004, P.R. China
}

Received July 29, 2016; Accepted December 28, 2016

DOI: $10.3892 /$ or.2017.5364

\begin{abstract}
Thyroid cancer (TC) is a common malignancy of the endocrine system. Abnormal expression of tripartite motif-containing 29 (TRIM29) has been reported to promote tumorigenesis and predict poor prognosis in several human malignancies. The aim of this study was to assess the involvement of TRIM29 in the significance and prognosis of TC. Fifty-six tumor samples and their clinicopathological parameters were obtained from TC patients; the expression level of TRIM29 was detected by RT-qPCR and western blotting. TRIM29 expression was knocked down by small interfering RNA (siRNA) among TT, TPC-1, and K1 cells to investigate the biological role of TRIM29 in TC cells. The results showed that TRIM29 expression was significantly increased in TC tissue samples and cells compared to normal tissues and cells ( $\mathrm{P}<0.01$, respectively). Overexpression of TRIM29 was associated with TNM stage $(\mathrm{P}<0.01)$, extrathyroidal extension $(\mathrm{P}<0.01)$, lymph node metastasis $(\mathrm{P}<0.05)$, and distant metastasis $(\mathrm{P}<0.05)$. Furthermore, the overall survival and disease-free rates of patients with high TRIM29 expression were decreased significantly compared with those with low TRIM29 expression ( $\mathrm{P}<0.01$, respectively). Knockdown of TRIM29 obviously suppressed cell proliferation; enhanced chemosensitivity to cisplatin; inhibited cell invasion and migration; caused cell cycle arrest at G0/G1 phase by decreasing cyclin B1, cyclin D1 and CDK2, while increasing p21 and p27; and induced cell apoptosis by enhancing the activities of caspase-3, caspase-9, and Bax, while decreased Bcl-2. Notably, decreased TRIM29 expression significantly inhibited the activation of P13K/AKT signaling pathway as well. Taken together, our findings suggested that TRIM29 played a crucial role in the progression and malignancy of TC, and silencing of
\end{abstract}

Correspondence to: Dr JianCang Ma, Department of General Surgery, The Second Affiliated Hospital of Xi'an Jiaotong University, Xi'an, Shaanxi 710004, P.R. China

E-mail: majiancangery@163.com

Key words: TRIM29, thyroid cancer, poor prognosis, apoptosis, cell cycle arrest, P13K/AKT signaling pathway
TRIM29 exerted its antitumor effect by blocking P13K/AKT signaling pathway. Thus, TRIM29 might be a potential therapeutic target for the treatment of TC.

\section{Introduction}

Thyroid cancer (TC) is the most common malignancy of the endocrine system, and the incidence of TC rapidly increases worldwide each year. Thyroid carcinoma can be divided into papillary, follicular, medullary and anaplastic types according to histological type (1). Papillary is the most common thyroid carcinoma, accounting for $>83 \%$ of all such malignancies (2). In recent years, the prognosis of TC patients has shown improvement due to advances in chemotherapy and radiotherapy. However, growing evidence demonstrates that patients with advanced TC are resistant to uptake of radioiodine or surgical resection, which leads to the disease recurrence or even death (3). Therefore, it is urgent to investigate novel strategies for the diagnosis and treatment of TC in early stage.

Tripartite motif-containing 29 (TRIM29), also known as ataxia-telangiectasia group D-associated protein (ATDC), belongs to the TRIM family, TRIM family is composed of evolutionary conserved N-terminal tripartite motif, such as B-box, RING finger and coiled-coil (RBCC) domain $(4,5)$. The TRIM family members have been proved to be involved in various physiological and biological processes including cell apoptosis, proliferation and oncogenesis, once altered, pathological conditions will occur (6). The structure of TRIM29 is dissimilar to other TRIM protein members, it has no RING domain, indicating no E3 ubiquitin ligase activity of TRIM29 functions. Recent studies have reported that overexpression of TRIM 29 is associated with tumor progression and poor prognosis in a variety of cancers, such as lung cancer, gastric cancer, pancreatic adenocarcinoma, bladder cancer, ovarian cancer and endometrial neoplasms (7-9). Liu et al have verified that TRIM29 knockdown inhibited cell proliferation and invasion in human squamous cancer cells (10). Moreover, in colorectal cancer, upregulation of TRIM29 is associated with advanced tumor stage, histological differentiation, and predicts poor survival (11). In contrast, the tumor suppressor role of TRIM29 is also reported in prostate and breast cancer $(12,13)$. However, the biological function and molecular details in TC progression has not been well established. 
In the present study, to illustrate the underlying molecular mechanisms of TRIM29 in TC development, TRIM29 expression level was evaluated in TC tumor samples and cell lines. We found that overexpression of TRIM29 was correlated with clinicopathological parameters and poor prognosis in TC patients. TRIM29 knocking down by siRNA method led to the inhibition of cell proliferation by arresting the cell cycle and inducing cell apoptosis, and further confirmed that the P13K/AKT signaling pathway was involved in the mediation of TRIM29 in TC development.

\section{Materials and methods}

Collection of tissue samples. In this study, 56 thyroid cancer (TC) tissues and paired adjacent normal tissues were obtained from TC patients who underwent total thyroidectomy at the Department of General Surgery, the Second Affiliated Hospital of Xi'an Jiaotong University, Xi'an, China, between November 2009 and September 2014. All TC samples were separately diagnosed and confirmed by two pathologists. Tumor stage was performed according to the sixth edition of the TNM (tumor, node, and metastasis) classification of the International Union Against Cancer. The clinical characteristics of patients including gender, age, TNM stage, tumor size, extrathyroidal extension, regional lymph nodes and distant metastasis were collected. This study was approved by the Ethics Committee of the Second Affiliated Hospital of Xi'an Jiaotong University and accordance with the Declaration of Helsinki, and the informed consents were obtained from all patients. The expression level of TRIM29 in tissue samples lower than the median level were considered as the low TRIM29 expression group and vice versa. A five-year survival time and outcomes were recorded in follow-up. The median duration of follow-up was 38 months (range: 1-60 months).

Cell line and cell culture. The human TC cell lines TT, TPC-1, $\mathrm{K} 1$, and human normal thyroid cell line Nthy-Ori-3-1 were purchased from the Cell Bank of the Chinese Academy of Medical Science (Beijing, China). All cells were cultured in DMEM medium (Invitrogen, Carlsbad, CA, USA) supplemented with $10 \%$ fetal bovine serum (Gibco, Carlsbad, CA, USA) and $1 \%$ penicillin/streptomycin under an atmosphere of $5 \% \mathrm{CO}_{2}$ at $37^{\circ} \mathrm{C}$.

Silencing TRIM29 expression in TC cells by siRNA. The siRNA (small interfence RNA) method was employed to knock down TRIM29 expression in TC cell lines. The sequences for siRNA were design as follows: sense strand 5'-AGU AGU UGG AGU UCU UGU CGU-3', antisense strand 5'-GAC AAG AAC UCC AAC UAC UUC-3'. The scramble siRNA was random sequenced by Blast website. Then the recombinant plasmids (si-TRIM29) or scramble siRNA (si-NC) was transfected into TC cells using Lipofectamine 2000 (Invitrogen) according to the manufacturer's instructions. The efficacy of siRNA knockdown was determined by RT-qPCR and western blotting.

Real-time quantitative PCR analysis. Total RNA was extracted from either tissue samples or cells using TRIzol (Invitrogen) according to the manufacturer's protocol, and then the isolated mRNA were reversely transcribed into cDNA by
M-MLV Reverse transcriptase kit (Promega, Madison, WI, USA) according to the manufacturer's protocol. RT-qPCR analysis was performed by SYBR-Green Master Mix kit (Takara, Otsu, Japan). The primer sequences were as follows: TRIM29 sense, 5'-ATG CTT GGT GGT CAC TTT GG-3' and antisense, 5'-GCA CTT CCC TTA CCA GCA TAG-3'; $\beta$-actin sense, 5'-CAG CAT CAT GAA GTG CGA CG-3' and antisense, 5'- CTG CAG TGT GGT GCT TCA AC-3'. The mRNA expression of TRIM29 was normalized to the housekeeping gene $\beta$-actin, which was used as an internal control. In brief, the PCR conditions were as follows: $95^{\circ} \mathrm{C}$ for $3 \mathrm{~min}, 95^{\circ} \mathrm{C}$ for $15 \mathrm{sec}, 65^{\circ} \mathrm{C}$ for $30 \mathrm{sec}$ for 40 cycles. The relative quantification referred as relative expression level was calculated by the value of $2^{-\Delta \Delta C t}$. Each experiment was performed in triplicate.

Western blot analysis. Tissue samples or cells were washed twice with ice-cold PBS and lysed with RIPA lysis buffer (Beyotime, Haimen, China). BCA assay was used to quantify the protein concentration of cell lysates. Total proteins were separated by $15 \%$ SDS-polyacrylamide (SDS-PAGE) and then transferred onto PVDF membranes (Pierce, Thermo Scientific, Rockford, IL, USA). After blocking with 5\% BSA for $1 \mathrm{~h}$, the membranes were incubated with appropriate primary antibodies at $4^{\circ} \mathrm{C}$ overnight. Next, the membranes were incubated with goat anti-rabbit HRP-conjugated secondary antibody (diluted at 1:2000) (Santa Cruz Biotechnology) at $37^{\circ} \mathrm{C}$ for $1 \mathrm{~h}$. The protein bands were detected using the Enhanced Chemiluminescence Detection System (Pierce, Thermo Scientific). $\beta$-actin was used for normalization and the protein intensity was quantified by using Image-Pro Plus 6.0 software (Media Cybernetics, MD, USA).

Cell viability assay. Cell proliferation was determined by the MTT [3-(4,5-dimethylthiazol-2-yl)-2,5-diphenyltetrazolium bromide] (Beyotime) assay according to the manufacturer's protocol. Cells were seeded in 96-well plates at the density of $5 \times 10^{3}$ cells/well after transfected with siRNA-TRIM29 or siRNA-NC for $48 \mathrm{~h}$ and incubate at $37^{\circ} \mathrm{C}, 5 \% \mathrm{CO}_{2}$ for 5 days. For chemotherapy drug sensitivity test, the transfected cells were incubated with cisplatin (Sigma-Aldrich, St. Louis, MO, USA) at the doses of $0,5,10,15,20 \mu \mathrm{g} / \mathrm{ml}$ for $24 \mathrm{~h}$. At indicated time points, $20 \mu \mathrm{l}$ MTT solution $(5 \mathrm{mg} / \mathrm{ml})$ was added into each well and incubated for $4 \mathrm{~h}$ at $37^{\circ} \mathrm{C}$. Dimethyl sulfoxide (DMSO) $(150 \mu \mathrm{l})$ was added into the well to dissolve the crystals for 10 min on a low-speed shaker. The OD (optical density) value was measured each day at $490 \mathrm{~nm}$ using a microplate reader (BioTek, Winooski, VT, USA).

Colony formation assay. Cells were seeded at the density of 500 cells/well in 6-well plate after transfected with siRNATRIM29 or siRNA-NC for $48 \mathrm{~h}$, and then incubated at $37^{\circ} \mathrm{C}$, $5 \% \mathrm{CO}_{2}$ for 14 days. Colonies were fixed with $95 \%$ ethanol for 10 min after washing with PBS twice, and then stained with crystal violet for $20 \mathrm{~min}$, colonies with at least 50 cells were counted and photographed. Each experiment was performed in triplicate.

Cell cycle analysis. Cells were harvested after transfected with siRNA-TRIM29 or siRNA-NC for $48 \mathrm{~h}$ and fixed in $75 \%$ 

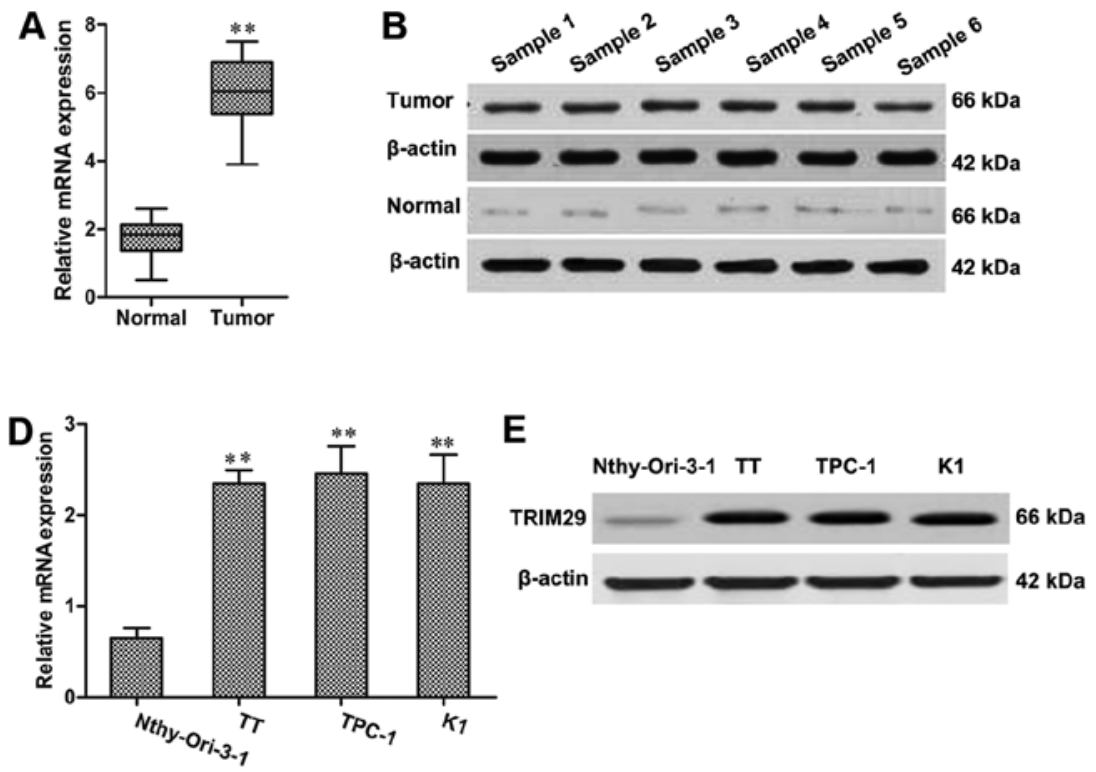
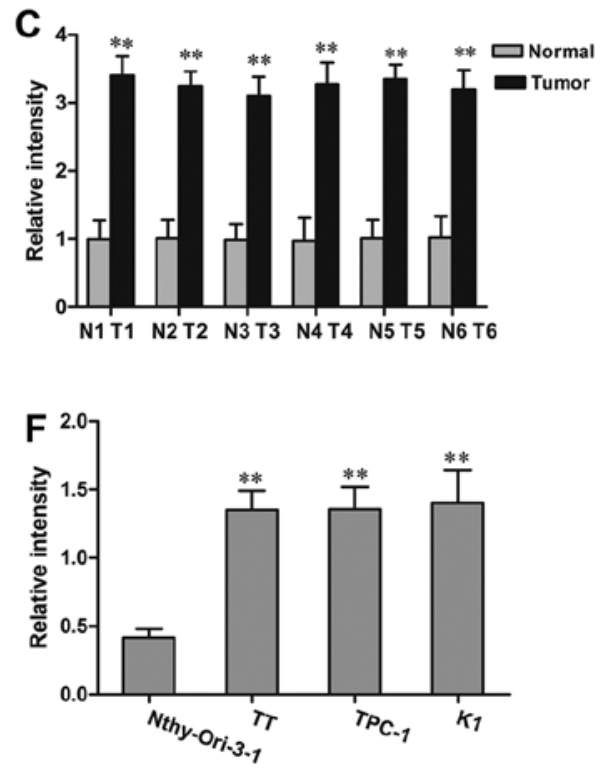

Figure 1. Expression of TRIM29 mRNA and protein in TC tissues and cell lines. (A) The relative level of TRIM29 mRNA in TC tissues and adjacent normal tissues was detected by RT-qPCR. (B) Representative expression level of TRIM29 protein in TC tissues and adjacent normal tissues was detected by western blotting. (C) The relative level of TRIM29 protein described in (B) was counted by $\beta$-actin as normalization. The relative levels of TRIM29 mRNA (D) and protein (E) in TC cell lines TT, TPC-1, K1, and human normal thyroid cell line Nthy-Ori-3-1 were detected by RT-qPCR and western blotting. (F) The relative level of TRIM29 protein described in (E) was counted by $\beta$-actin as normalization. Data are shown as mean \pm SD, all the experiments were in triplicate. ${ }^{* *} \mathrm{P}<0.01$ means significant, when compared to control group.

ethanol overnight, and then washed with PBS, stained by propidium iodide $(5 \mathrm{mg} / \mathrm{ml})$ containing RNase A. Cell cycle distributions were analyzed by flow cytometer FACSCalibur (Beckman Coulter, Inc., Fullerton, CA, USA) according to the manufacturer's protocol. Each experiment was performed in triplicate.

Cell apoptosis analysis. Cells were harvested after transfected with siRNA-TRIM29 or siRNA-NC for $48 \mathrm{~h}$, and then washed with cold PBS, and stained with Annexin V-FITC double staining kit (BD Bioscience, San Jose, CA, USA) and analyzed using flow cyctometry FACSCalibur (Beckman Coulter, Inc.) according to the manufacturer's protocol. Each experiment was performed in triplicate.

Cell migration and invasion assay. Cells were harvested after transfected with siRNA-TRIM29 or siRNA-NC for $48 \mathrm{~h}$, and then suspended with serum-free medium, then seeded in the upper chamber of Transwell $(8 \mu \mathrm{m}$ pore size, Corning Inc., Coring, NY, USA) at the density of $5 \times 10^{4}$ cells per chamber for invasion assay and $1 \times 10^{5}$ cells per chamber for migration assay, respectively. For invasion assay, the upper chambers were precoated with Matrigel (BD Bioscience). The lower chambers were filled with medium containing $10 \%$ FBS and incubated for $24 \mathrm{~h}$. Cells remaining on the upper chamber were removed carefully, the migrating cells in the lower chamber were fixed with $100 \%$ methanol, and stained with crystal violet. The stained cells were imaged with an inverted microscope (Olympus), and counted at ten randomly selected visual fields for each experimental condition. Each experiment was performed in triplicate.

Statistical analysis. Statistical analyses were performed using SPSS-12.0 software. Each experiment was performed in triplicate, unless otherwise indicated. All data are presented as mean \pm standard deviation (SD). Comparisons between groups were done using the unpaired Student's t-test. A $\mathrm{P}$-value of $<0.05$ was considered to indicate a statistically significant difference.

\section{Results}

Elevated expression of TRIM29 in TC tissue samples and cell lines. RT-qPCR and western blot assays were used to detect the expression of TRIM29 in TC tissue samples and adjacent paired normal tissues. As shown in Fig. 1A, the mRNA expression of TRIM29 was significantly upregulated in TC tissue samples compared with the adjacent paired normal tissues. Consistent with RT-qPCR results, the western blot also revealed that TRIM29 was upregulated at protein levels in TC samples (Fig. 1B and C). Furthermore, the expression level of TRIM29 was evaluated among three TC cell lines (TT, TPC-1, and K1) and normal thyroid cell line Nthy-Ori-3-1. The result showed that TRIM29 expression was remarkably increased in TT, TPC-1, and K1 cells when compared to Nthy-Ori-3-1 cell line (Fig. 1D-F).

TRIM29 expression correlates with clinicopathological parameters on the prognosis of TC patients. The correlation between TRIM29 expression and clinicopathological parameters of all patients were investigated by Peason's Chi-square analysis. TC cases were subdivided into two groups according to the mRNA expression level of TRIM29 as described above. High TRIM29 expression was observed in $73.21 \%$ (41/56) of cases, while $26.79 \%$ (15/56) had low TRIM29 expression. The high TRIM29 expression was associated with TNM stage $(\mathrm{P}<0.01)$, extrathyroidal extension $(\mathrm{P}<0.01)$, lymph nodes metastasis $(\mathrm{P}<0.05)$, and distant metastasis $(\mathrm{P}<0.05)$, 

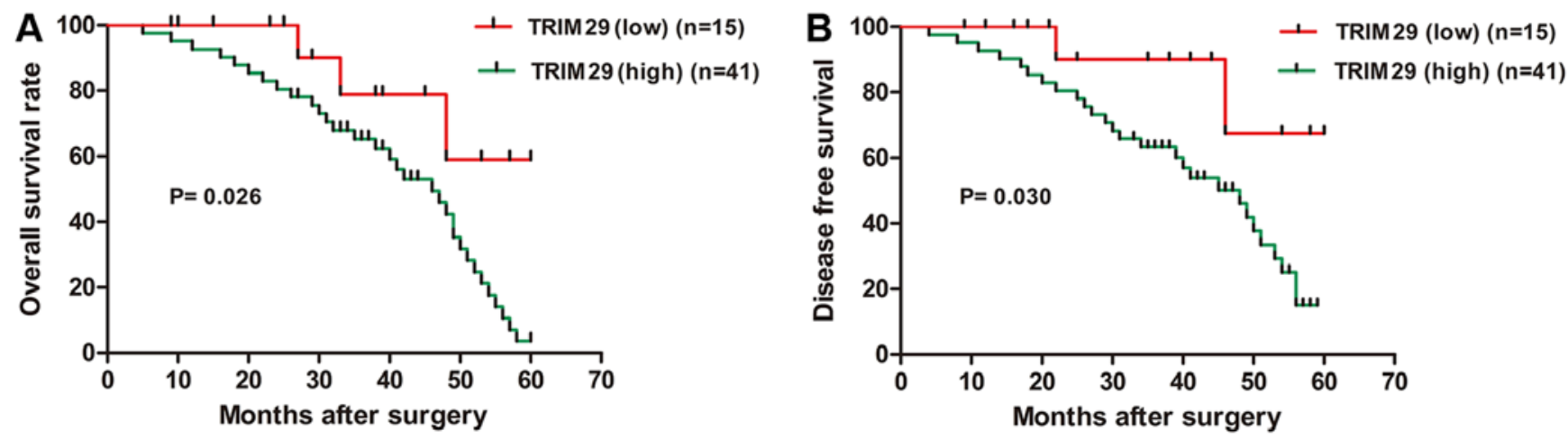

Figure 2. Kaplan-Meier curves for (A) overall survival (OS) and (B) disease-free survival (DFS) in 56 TC patients according to TRIM29 positive (+) or TRIM29 (-) negative expression. ${ }^{*} \mathrm{P}<0.05$ was considered statistically significant.

but had no correlation with patient gender, age or tumor size (Table I). In addition, the survival time of the two groups was compared by Kaplan-Meier analysis, the cumulative 5-year overall survival rate (OS) was $21.95 \%$ in the high TRIM29 expression group whereas $80.00 \%$ in the low TRIM29 expression group $(\mathrm{P}<0.05)$; while the disease-free survival rate (DFS) was $34.15 \%$ in the high TRIM29 expression group which was lower than $86.67 \%$ in the low TRIM29 expression group $(\mathrm{P}<0.05)$ (Fig. 2). These findings demonstrated that the TRIM29 overexpression was correlated with poor prognosis in TC patients.

Knockdown of TRIM29 expression in TC cells inhibits cell proliferation. The expression of TRIM29 was knocked down in TT, TPC-1, and K1 cells by using siRNA method. The efficiency of TRIM29 knockdown was confirmed by RT-qPCR and western blotting (Fig. 3A). Next, the viability of cells with TRIM29 knockdown was detected by MTT assay. As shown in Fig. 3B, TRIM29 knockdown significantly decreased the viability of TT, TPC-1, and $\mathrm{K} 1$ cells $(\mathrm{P}<0.01$, respectively) when compared with NC group. Furthermore, in agreement with MTT data, colony formation assays also illustrated that the numbers of colonies were significantly reduced in TT, TPC-1, and K1 cells with TRIM29 knockdown compared to corresponding NC groups $(\mathrm{P}<0.01$, respectively; Fig. 3C). These results suggested that downregulated TRIM29 expression inhibited the proliferation of TC cells.

TRIM29 knockdown enhanced the sensitivity of TC cells to chemotherapy. The effect of TRIM29 knockdown on the sensitivity of TC cells to cisplain was determined by MTT method. We transfected TT, TPC-1, and K1 cells with siRNA-TRIM29 or siRNA-NC and treated with cisplatin at various doses $(0$, $5,10,15,20 \mu \mathrm{g} / \mathrm{ml}$ ) for $24 \mathrm{~h}$. As shown in Fig. 3D, compared with the control groups, TT, TPC-1, and K1 cells with siRNATRIM29 transfection significantly decreased cell viability by enhancing cell sensitivity to cisplatin under different-dose treatments $(10,15,20 \mu \mathrm{g} / \mathrm{ml}$, respectively). Thus, we concluded that the downregulation of TRIM29 by siRNA-TRIM29 enhanced cell sensitivity to cisplatin in TC development.

Silencing of TRIM29 expression reduces invasion and migration of TC cells. To investigate whether the knockdown of TRIM29 influences invasion and migration of TC cells,
Table I. TRIM29 expression and clinicopathological factors in TC patients.

\begin{tabular}{|c|c|c|c|c|}
\hline \multirow[b]{2}{*}{ Variables } & \multirow[b]{2}{*}{ Case } & \multicolumn{2}{|c|}{ TRIM29 expression } & \multirow[b]{2}{*}{ P-value } \\
\hline & & $\begin{array}{c}\text { Low } \\
(n=15)\end{array}$ & $\begin{array}{c}\text { High } \\
(n=41)\end{array}$ & \\
\hline Age & 56 & 15 & 41 & 0.431 \\
\hline$>45$ & 28 & 8 & 20 & \\
\hline$\leq 45$ & 28 & 7 & 21 & \\
\hline Gender & & 15 & 41 & 0.708 \\
\hline Male & 30 & 10 & 20 & \\
\hline Female & 26 & 5 & 21 & \\
\hline Tumor size & & & & 0.528 \\
\hline$>2$ & 25 & 3 & 22 & \\
\hline$\leq 2$ & 31 & 12 & 19 & \\
\hline \multicolumn{2}{|c|}{ Extrathyroidal extension } & & & $<0.01^{\mathrm{a}}$ \\
\hline Negative & 23 & 10 & 13 & \\
\hline Positive & 33 & 5 & 28 & \\
\hline \multicolumn{2}{|c|}{ Lymph node metastasis } & & & $<0.05^{\mathrm{a}}$ \\
\hline Absent & 26 & 5 & 21 & \\
\hline Present & 30 & 10 & 20 & \\
\hline \multicolumn{2}{|c|}{ Distant metastasis } & & & $<0.05^{\mathrm{a}}$ \\
\hline Negative & 29 & 8 & 21 & \\
\hline Positive & 27 & 7 & 20 & \\
\hline TNM stage & & & & $<0.01^{\mathrm{a}}$ \\
\hline $\mathrm{I} / \mathrm{II}$ & 27 & 6 & 21 & \\
\hline III/IV & 29 & 9 & 20 & \\
\hline
\end{tabular}

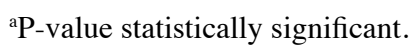

Transwell invasion and migration assays were conducted. As shown in Fig. 4A, the numbers of invading cells in TT, TPC-1, and K1 cells with TRIM29 knockdown were obviously reduced compared to control groups ( $\mathrm{P}<0.01$, respectively). Consistent with invasion assay, migration assay (Fig. 4B) also revealed that, 
A

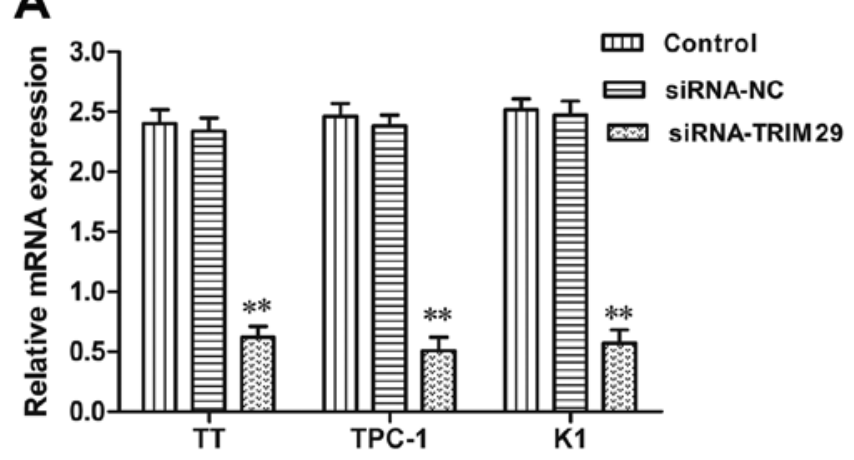

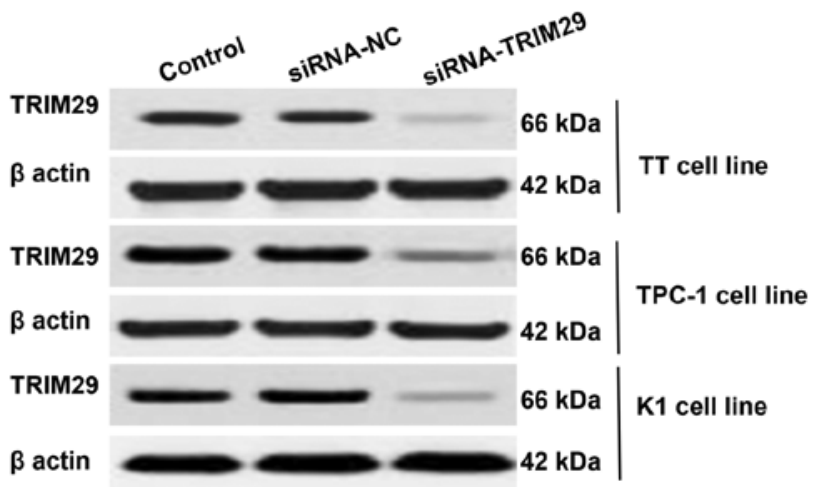

TPC-1 cell line
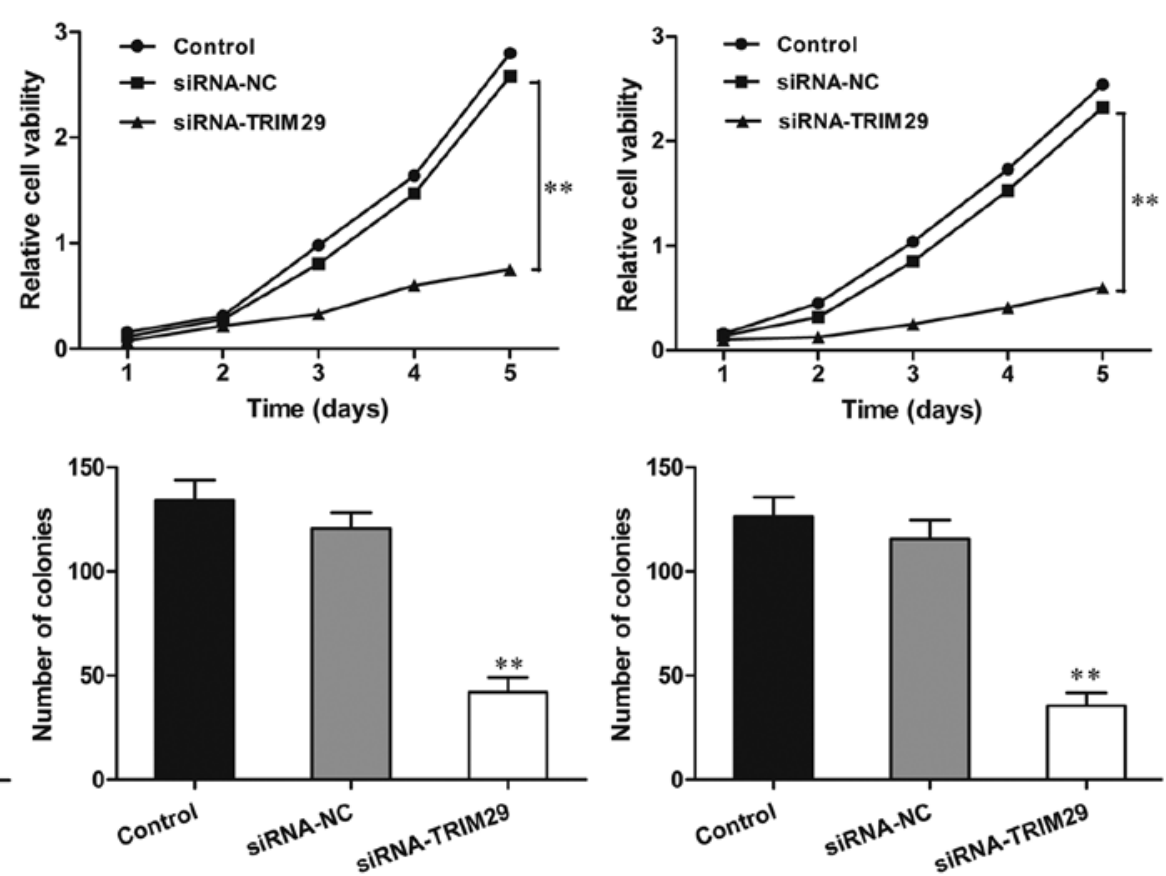

TPC-1 cell line

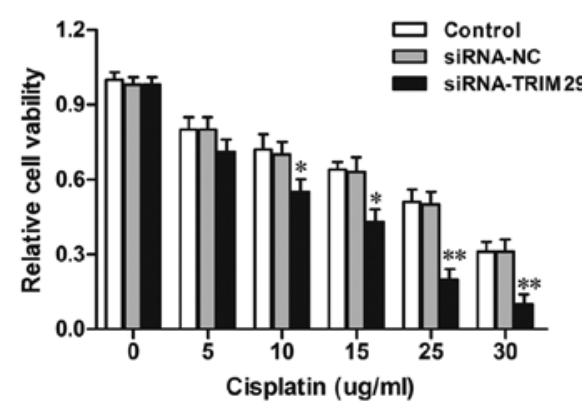

K1 cell line

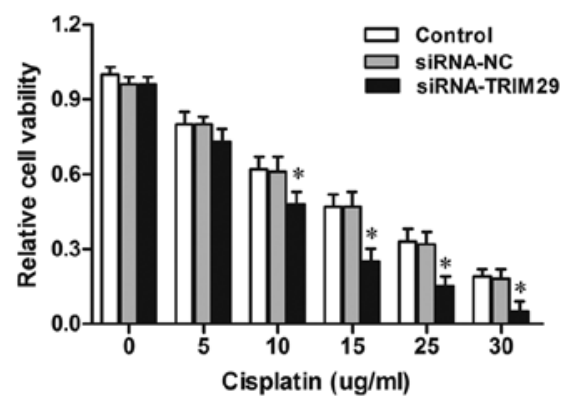

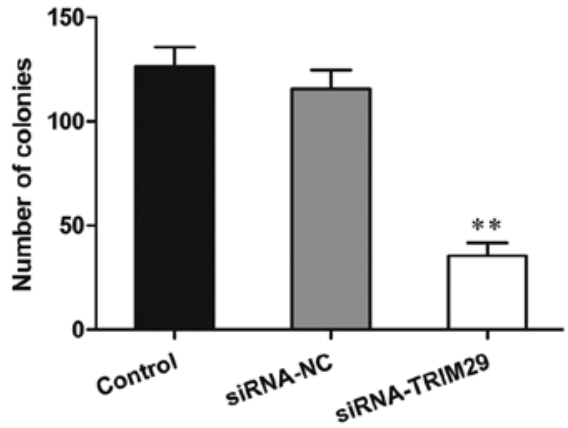

Figure 3. The inhibition of cell proliferation and enhancement of chemosensitivity on TC cells with TRIM29 knockdown. (A) Identification of TRIM29 knockdown in TC cell lines. (B) Growth curve of TC cells with TRIM29 knockdown was examed by MTT assay. (C) The number of colonies in TC cells with TRIM29 knockdown was detected by colony formation assay. (D) Cell viabilities of TC cells with TRIM29 knockdown and cisplatin treatments were detected by MTT assay. Data are shown as mean $\pm \mathrm{SD}$; all the experiments were in triplicate. ${ }^{* *} \mathrm{P}<0.01$, statistically significant, when compared to siRNA-NC group.

when compared with control group, the number of migrated cells were significantly decreased in TT, TPC-1, and K1 cells with TRIM29 knockdown ( $\mathrm{P}<0.01$, respectively), which indicating that downregulation of TRIM29 suppressed TC cell invasion and migration. To further address the role of TRIM29 knockdown in cell invasion and migration, western blot result revealed that TRIM29 knockdown significantly decreased the hallmarks of cell invasion and migration including matrix metallopeptidases (MMP2 and MMP9).

TRIM29 silencing causes cell cycle arrest at G0/G1 phases in $T C$ cells. The flow cytometry assay showed that knockdown of TRIM29 contributed to a remarkably increased in the G0/G1 phase in TT, TPC-1, and K1 cells compared to cells 

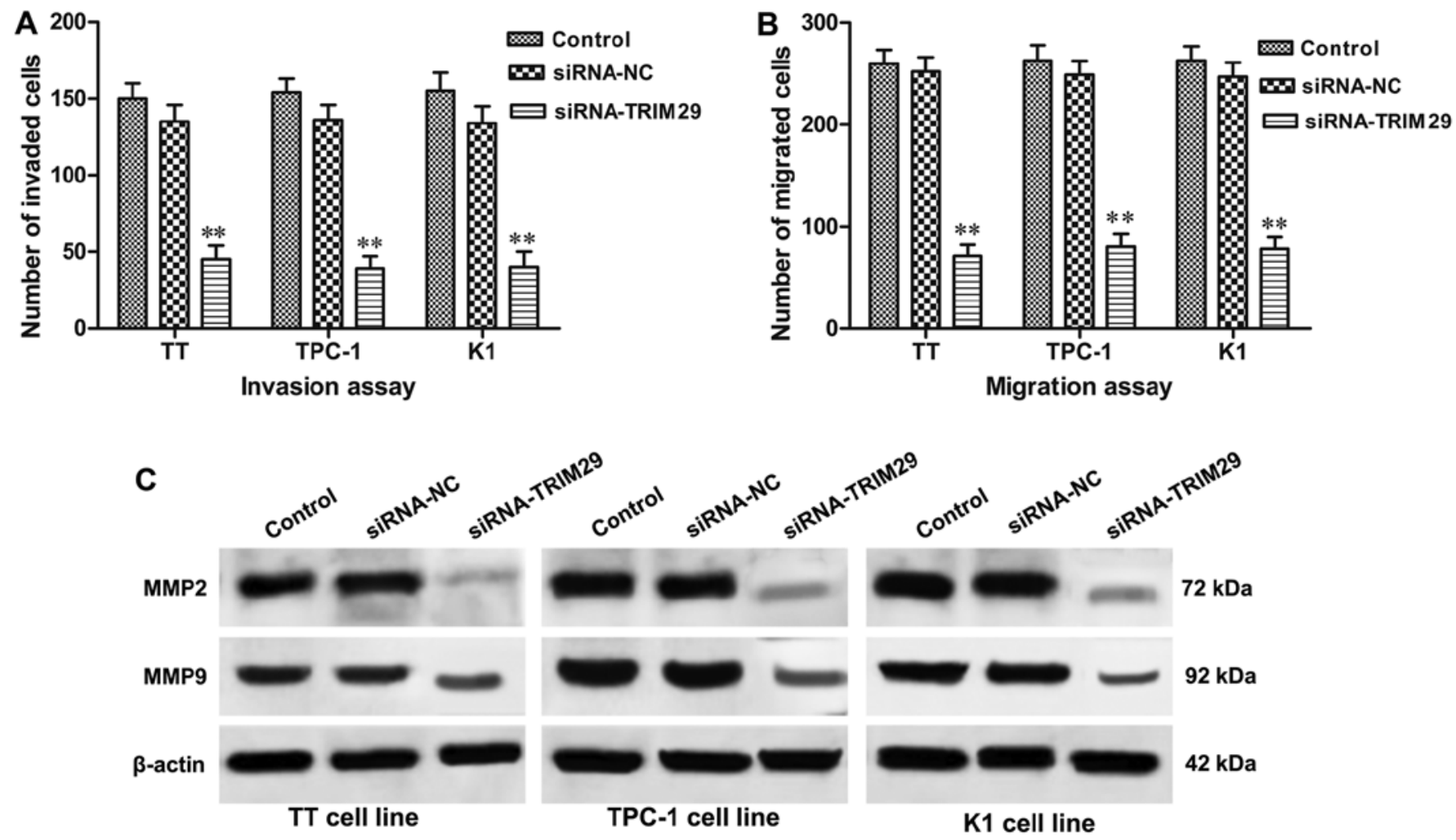

Figure 4. The inhibition of cell invasion and migration on TC cells with TRIM29 knockdown. (A) Number of invasive cells with siRNA transfection was detected by invasion assay. (B) Number of migrated cells with siRNA transfection was detected by migration assay. (C) Expression levels of MMP-2 and MMP-9 proteins in TC cells with siRNA transfection were detected by western blotting. Data are shown as mean \pm SD; all the experiments were in triplicate. ${ }^{* *} \mathrm{P}<0.01$, statistically significant, when compared to si-NC group.
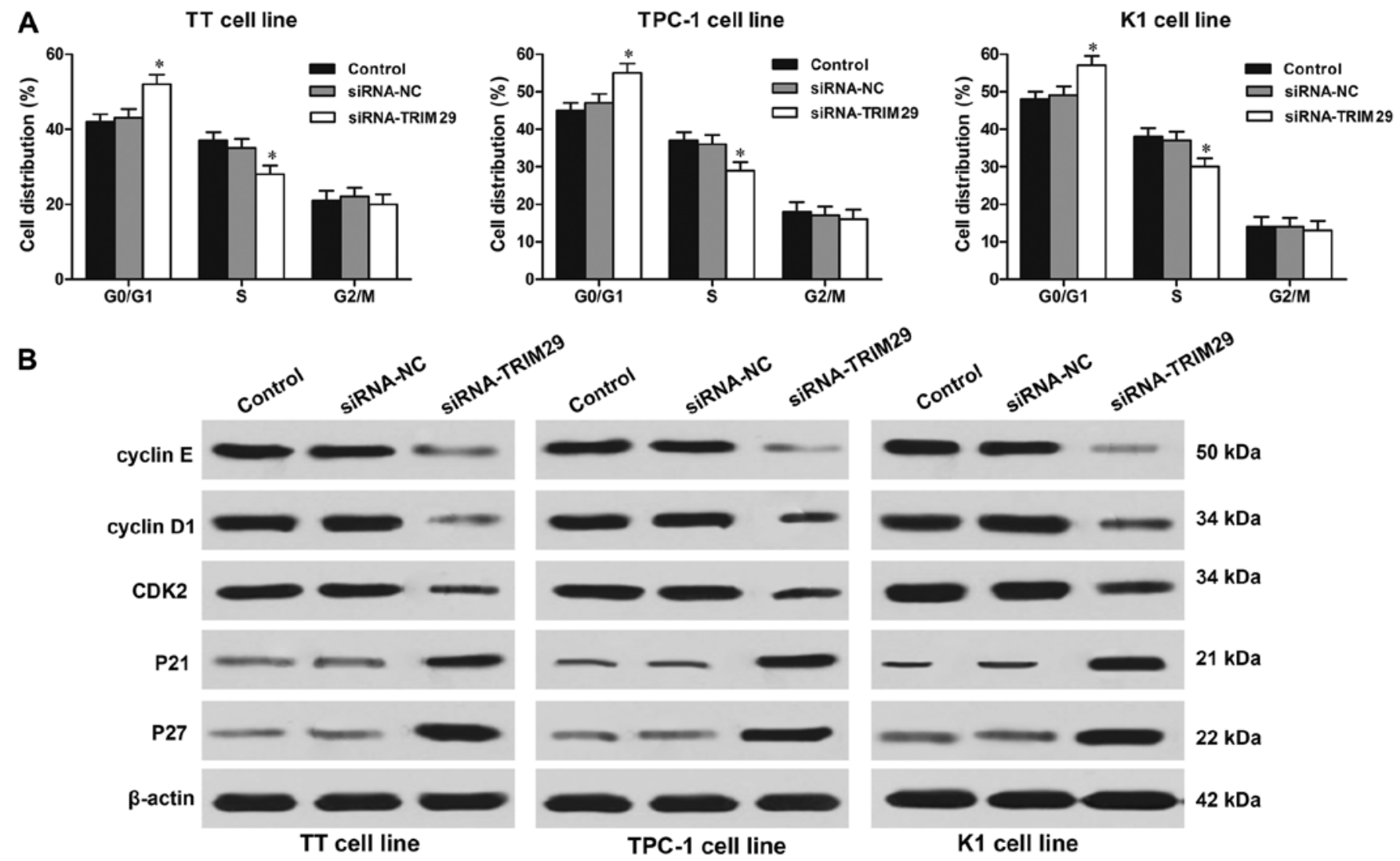

Figure 5. TRIM29 knockdown in TC cells delays the cell cycle. (A) Cell distribution analysis of TC cell cycle with TRIM29 knockdown. (B) The expression levels of related cell cycle regulatory proteins cyclin E, cyclin B1, CDK2, p21 and p27 were detected by western blotting. Data are shown as mean \pm SD; all the experiments were in triplicate. ${ }^{*} \mathrm{P}<0.05$, statistically significant, when compared to siRNA-NC group. 
A
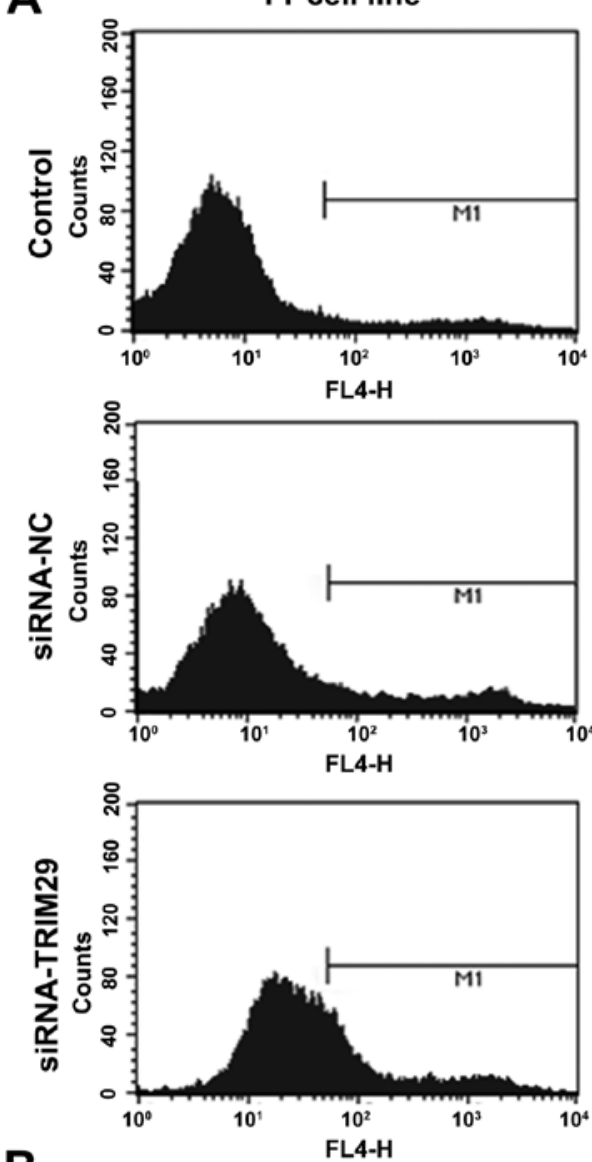

B

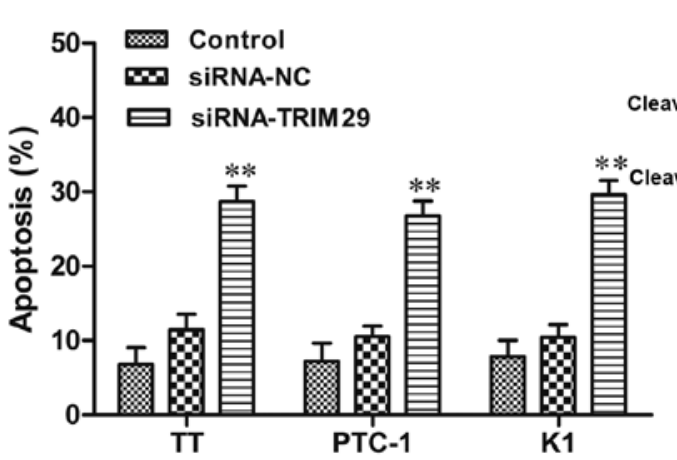

TPC-1 cell line
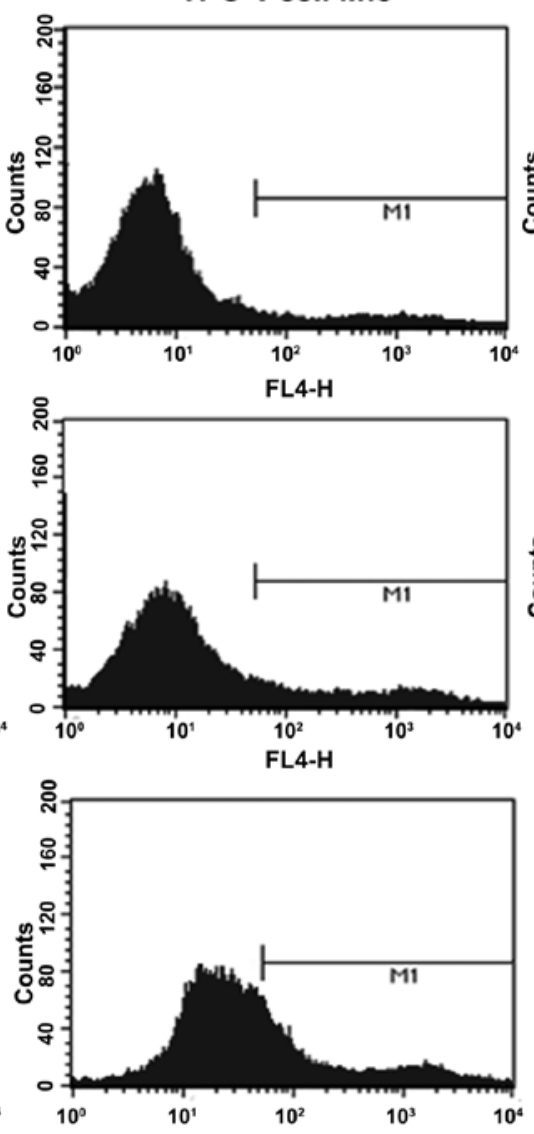

C
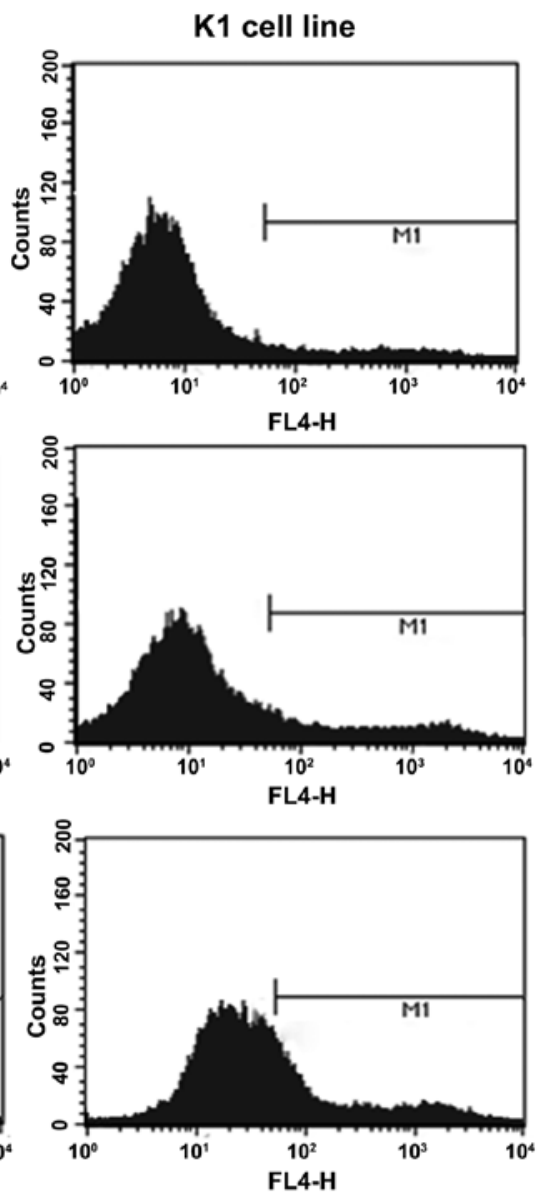
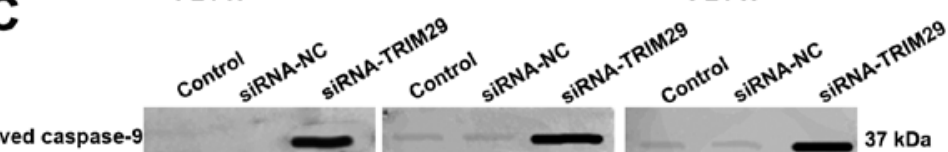

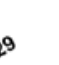

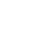


A

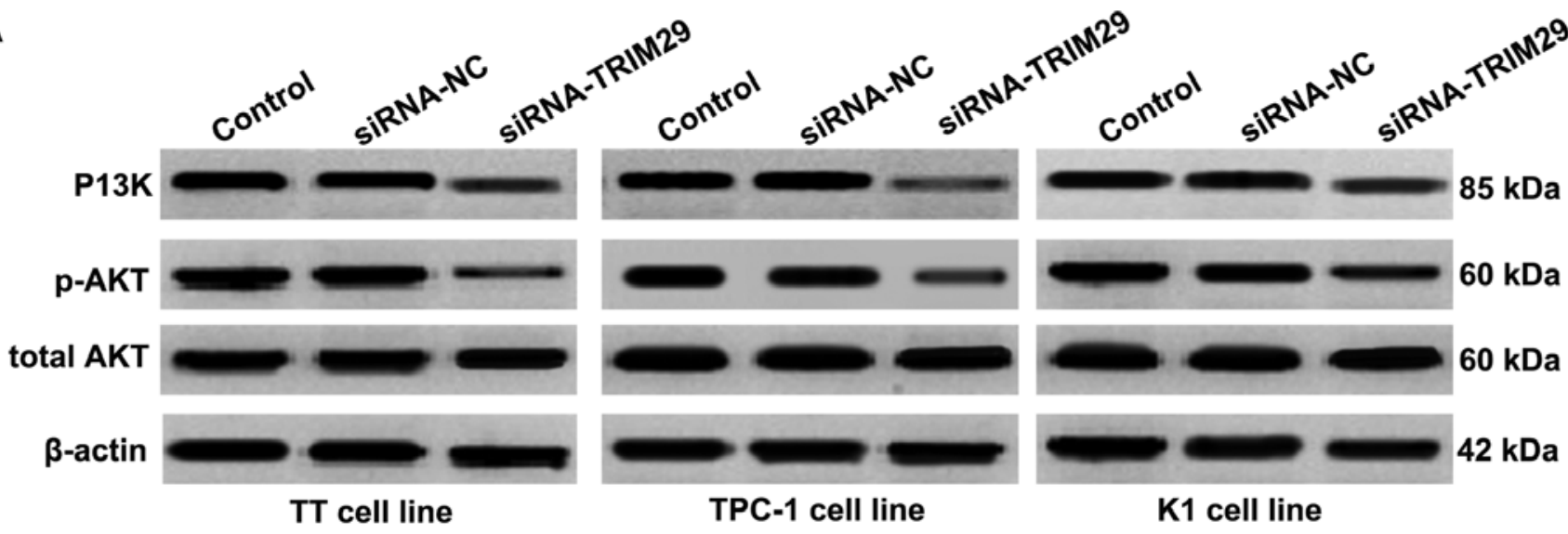

B

TT cell line

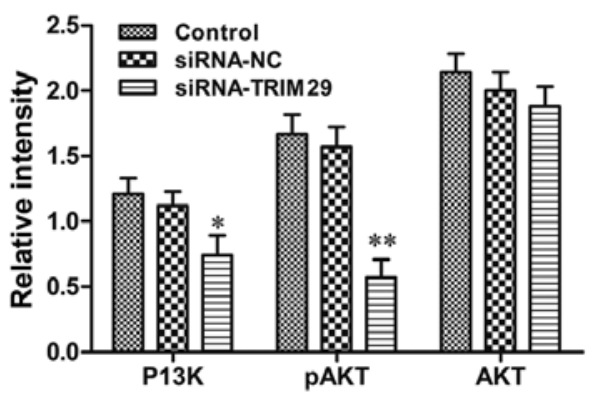

TPC-1 cell line

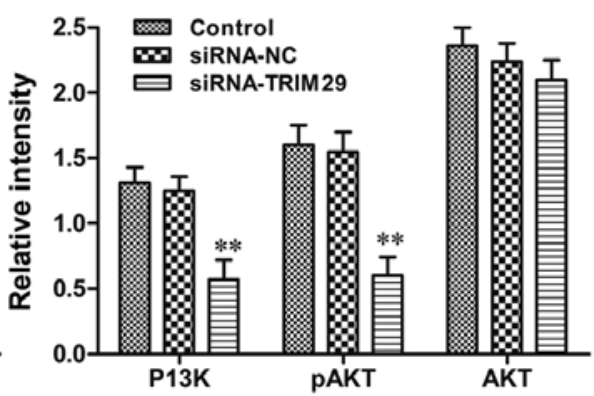

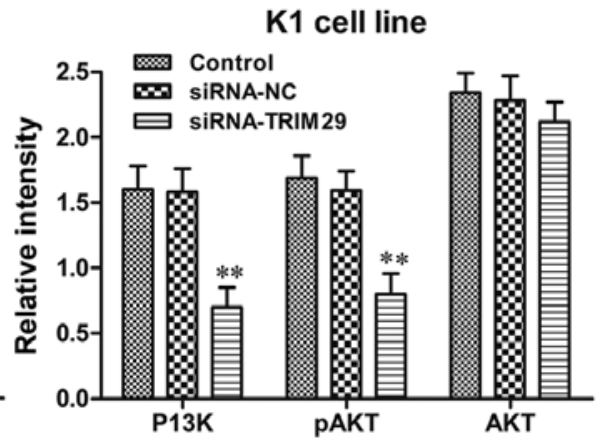

Figure 7. TRIM29 knockdown in TC cells inhibits the activation of P13K/AKT signaling pathway. (A) Western blot assay for the expression of key components of P13K, p-AKT, and total AKT of P13K/AKT signaling pathway. (B) The relative levels of proteins described in (B) were counted by $\beta$-actin as normalization. Data are shown as mean $\pm \mathrm{SD}$; all the experiments were in triplicate. ${ }^{*} \mathrm{P}<0.05,{ }^{* *} \mathrm{P}<0.01$, statistically significant, when compared to siRNA-NC group.

Knockdown of TRIM29 suppresses the P13K/AKT signaling pathway. The phosphoinositide 3 -kinase (P13K)/AKT signaling pathway plays a crucial role in thyroid oncogenesis and affects proliferation, metastasis, apoptosis of TC cells (14). Since the inhibitory effect of TRIM29 knockdown on TC cell cycle and apoptosis has been clarified, to further determine whether P13K/AKT signaling pathway is involved in TRIM29-mediated TC tumorigenesis, the key molecules of the P13K/AKT pathway were detected in TT, TPC-1, and K1 cells with TRIM29 knockdown. Western blot results showed that the $\mathrm{P} 13 \mathrm{~K}$, and phosphorylation of AKT expression levels were prominently decreased in TT, TPC-1, and K1 cells with downregulation of TRIM29 compared to vehicle-treated cells, though the total AKT levels were similar among different treated groups (Fig. 7). Our results illustrated that TRIM29 knockdown significantly inhibited the P13K/AKT signaling pathway in TC cells.

\section{Discussion}

$\mathrm{TC}$ is the most common endocrine malignancy, with increasing knowledge of its molecular pathogenesis $(15,16)$. In order to increase the efficacy of the therapeutic treatment and to reduce side effects, the investigations on gene dysregulation during TC progression are becoming the novel subject of research in this field. The location of TRIM29 is at chromosome 11q23, and functions as a sensitive component of a protein kinase mediated signaling transduction pathway against ionizing radiation, which might aggravate tumorigenesis and progression $(17,18)$. TRIM29 is involved in tumor proliferation and progression in a variety of cancer cells. However, among different tumor cell types, TRIM29 functioned either as tumor suppressor or oncogene according to the origin of the neoplasm (4). Recent analysis demonstrated that TRIM29 overexpression could promote tumor cell survival and growth in various types of cancers (19). To our knowledge, the investigation of the relationship between TRIM29 expression and TC tumorigenesis has not been previously reported.

In the present study, we found that TRIM29 mRNA and protein were significantly enhanced in TC tumor samples and cell lines, suggesting a pro-oncogenic role of TRIM29 in TC progression. Moreover, the high expression of TRIM29 was tightly associated with TNM staging, extrathyroidal extension, lymph nodes metastasis, and distant metastasis. Notably, the overall survival rate and disease-free rate of patients with high TRIM29 expression was much lower than those with low TRIM29 expression, indicating that TRIM29 might be a potential predictor for the prognosis of TC patients. These findings were in agreement with other reports on TRIM29 in many cancers. Kosaka et al reported that TRIM29 overexpression was correlated with the metastatic process and poor prognosis in gastric cancer patients (9).

A previous study emphasized that TRIM29 functioned as a histone-binding protein to assemble DNA repair 
proteins into chromatin and then activated DNA damage response (20). Wang et al also indicated that TRIM29 played a crucial role in DNA damage signaling and radioresistance in human pancreatic cancer cells (21). Xu et al demonstrated that TRIM29 was a novel prognostic biomarker for prediction in aggressive cervical cancer patients that need postoperative adjuvant chemoradiotherapy (22). Liu et al suggested that silencing of TRIM29 enhanced chemosensitivity to cisplatin in human lung squamous cancer by increasing cell apoptosis (10). Cisplatin is a widely used chemotherapeutic drug against various types of cancers through DNA damage mechanism (23). The present study stated the association of TRIM29 expression with cisplatin cell resistance in TC cells and proved knockdown of TRIM 29 made TC cells more sensitive to cisplatin treatment.

Studies have shown that TRIM29 is intimately associated with cell proliferation, cell cycle, invasion and cell apoptosis. Liu et al discovered that TRIM29 knockdown suppressed proliferation and invasion in human lung squamous cancer cells (10). Sun et al reported that TRIM29 promoted tumor cell proliferation and progression in pancreatic cancer (8). Yuan et al discovered that TRIM29 promoted cancer cell proliferation by suppressing p53 nulcear activities (24). To illustrate the function of TRIM29 on TC progression, siRNA approach was employed to silence TRIM29 expression in TC cell lines. Our data showed that TRIM29 knockdown significantly inhibited TC cell proliferation and reduced colony formation, caused cell cycle to halt at G0/G1 phase, accompanied by repressing the expression of cyclin D1, cyclin B1, and CDK2; while increasing the expression of p21 and p27, which resulted in cell growth inhibition. Cyclin D1 could phosphorylate $\mathrm{Rb}$ protein and accelerate cancer progression via G1-S phase of cell cycle (25). Furthermore, the activation of cyclin E-CDK2 complex has been proved as another crucial demand for the G1/S phase transition in cancer aggressiveness; conversely, p21 and p27 inhibit the activation of CDK-cyclin complexes during tumor development (26). Collectively, the alternations of cell cycle regulatory molecules may contribute to the phase distribution in TC cells.

In the present study, we found that the expression of TRIM29 was significant in lymph nodes metastasis, and distant metastasis in TC progression according to clinicopathological factor analysis. The abilities of invasion and metastasis of cancer cells are dependent on the degradation of the components of extracellular matrix and basement membranes, which is regulated by matrix metalloproteinase (MMP) family (27). Silencing of RTIM29 significantly reduced the invasive and migratory abilities of TC cells by inhibiting the activities of MMP-2 and MMP-9, which are implicated in dysregulation of tumor invasion and metastasis in numerous human malignancies (28). Furthermore, the imbalance between apoptosis-induced and -inhibited molecule expressions are responsible for the vulnerability of cells to apoptosis (29). Thus, in this study, the activation of caspase- 3 and caspase-9, decreased ratio of $\mathrm{Bax} / \mathrm{Bcl}-2$ indicated that TRIM29 knockdown induced TC cell apoptosis in a caspase- and Bax/ Bcl-2-dependent manner.

$\mathrm{P} 13 \mathrm{~K} / \mathrm{AKT}$ pathway is a vital intracellular signaling pathway for regulating various bioprocesses in mammalian cells, including cell proliferation, metabolism, transformation, motility and tumorigenesis (30). P13K/AKT pathway could induce the development of tumor and promote tumor metastasis via various pathways. In order to enhance cell survival, activation of $\mathrm{P} 13 \mathrm{~K} / \mathrm{AKT}$ pathway could in turn activate or inhibit a series of downstream target proteins, such as caspase-9, p21, Bax, MMP2, and mammalian target of rapamycin (mTOR) $(14,31,32)$. Tan et al proved that TRIM29 overexpression promoted cell proliferation and survival through NF- $\mathrm{B}$ signaling pathway in bladder cancer cells (19). $\mathrm{Xu}$ et al reported that TRIM29 overexpression enhanced tumor progression by activating $\mathrm{Wnt} / \beta$-actenin pathway in cervical cancer (22). Furthermore, a recent study demonstrated that elevated TRIM29 expression contributed to the cell proliferation and metastasis of nasopharyngeal cancer by activating PTEN/AKT/mTOR signaling pathway (33). On the basis of references above, in our study, we found that the antitumor effect of TRIM29 silencing was associated with the inhibition of P13K/AKT signaling pathway, which might be the pivotal mechanism of TRIM29 knockdown-mediated proliferation suppression, chemosensitivity enhancement, the inhibition of invasion and migration, delayed cell cycle transition, cell apoptosis in TC (34). However, the deep molecular details still remained to be further elucidated.

In conclusion, this study highlighted the crucial role of TRIM29 overexpression in TC prognosis and progression, and subsequently demonstrated that TRIM29 knockdown resulted in the alternations of cell proliferation, chemosensitivity to cisplatin, invasion and migration, cell cycle arrest, and cell apoptosis via blocking the activation of P13K/ AKT signaling pathway and the involved downstream target genes, which provide credible evidence for novel therapeutic target combined with the P13K/AKT signaling pathway for TC treatment.

\section{Acknowledgements}

We would like to thank Professor J.C. Ma for his intelligent suggestions.

\section{References}

1. Zhang X, Wang Z, Tian L, Xie J, Zou G and Jiang F: Increased expression of FGF19 contributes to tumor progression and cell motility of human thyroid cancer. Otolaryngol Head Neck Surg 154: 52-58, 2016.

2. Kong LL, Man DM, Wang T, Zhang GA and Cui W: siRNA targeting RBP2 inhibits expression, proliferation, tumorigenicity and invasion in thyroid carcinoma cells. Oncol Lett 10: 3393-3398, 2015.

3. Ye WC, Gao L, Huang J, Fang XM and Xie G: Suppressed Krüppel-like factor 17 expression induces tumor proliferation, metastasis and a poor prognosis in papillary thyroid carcinoma. Mol Med Rep 10: 2087-2092, 2014.

4. Hatakeyama S: TRIM proteins and cancer. Nat Rev Cancer 11: 792-804, 2011.

5. Napolitano LM and Meroni G: TRIM family: Pleiotropy and diversification through homomultimer and heteromultimer formation. IUBMB Life 64: 64-71, 2012.

6. Cambiaghi V, Giuliani V, Lombardi S, Marinelli C, Toffalorio F and Pelicci PG: TRIM proteins in cancer. Adv Exp Med Biol 770: 77-91, 2012.

7. Song X, Fu C, Yang X, Sun D, Zhang X and Zhang J: Tripartite motif-containing 29 as a novel biomarker in non-small cell lung cancer. Oncol Lett 10: 2283-2288, 2015.

8. Sun H, Dai X and Han B: TRIM29 as a novel biomarker in pancreatic adenocarcinoma. Dis Markers 2014: 317817, 2014. 
9. Kosaka Y, Inoue H, Ohmachi T, Yokoe T, Matsumoto T, Mimori K, Tanaka F, Watanabe $M$ and Mori M: Tripartite motif-containing 29 (TRIM29) is a novel marker for lymph node metastasis in gastric cancer. Ann Surg Oncol 14: 2543-2549, 2007.

10. Liu C, Huang X, Hou S, Hu B and Li H: Silencing of tripartite motif (TRIM) 29 inhibits proliferation and invasion and increases chemosensitivity to cisplatin in human lung squamous cancer NCI-H520 cells. Thorac Cancer 6: 31-37, 2015.

11. Jiang T, Tang HM, Lu S, Yan DW, Yang YX and Peng ZH: Up-regulation of tripartite motif-containing 29 promotes cancer cell proliferation and predicts poor survival in colorectal cancer. Med Oncol 30: 715, 2013

12. Ai L, Kim WJ, Alpay M, Tang M, Pardo CE, Hatakeyama S, May WS, Kladde MP, Heldermon CD, Siegel EM, et al: TRIM29 suppresses TWIST1 and invasive breast cancer behavior. Cancer Res 74: 4875-4887, 2014.

13. Kanno Y, Watanabe M, Kimura T, Nonomura K, Tanaka S and Hatakeyama S: TRIM29 as a novel prostate basal cell marker for diagnosis of prostate cancer. Acta Histochem 116: 708-712, 2014.

14. Jin S, Borkhuu O, Bao W and Yang YT: Signaling pathways in thyroid cancer and their therapeutic implications. J Clin Med Res 8: 284-296, 2016.

15. Yang X, Liu G, Xiao H, Yu F, Xiang X, Lu Y, Li W, Liu X, Li S and Shi Y: TPX2 overexpression in medullary thyroid carcinoma mediates TT cell proliferation. Pathol Oncol Res 20: 641-648, 2014.

16. Liu Z, Yang L, Teng X, Zhang H and Guan H: The involvement of CXCR7 in modulating the progression of papillary thyroid carcinoma. J Surg Res 191: 379-388, 2014.

17. Reymond A, Meroni G, Fantozzi A, Merla G, Cairo S, Luzi L, Riganelli D, Zanaria E, Messali S, Cainarca S, et al: The tripartite motif family identifies cell compartments. EMBO J 20: 2140-2151, 2001.

18. Kapp LN, Painter RB, Yu LC, van Loon N, Richard CW III, James MR, Cox DR and Murnane JP: Cloning of a candidate gene for ataxia-telangiectasia group D. Am J Hum Genet 51: 45-54, 1992.

19. Tan ST, Liu SY and Wu B: TRIM29 Overexpression promotes proliferation and survival of bladder cancer cells through NF- $\mathrm{B}$ signaling. Cancer Res Treat 48: 1302-1312, 2016.

20. Masuda Y, Takahashi H, Sato S, Tomomori-Sato C, Saraf A, Washburn MP, Florens L, Conaway RC, Conaway JW and Hatakeyama S: TRIM29 regulates the assembly of DNA repair proteins into damaged chromatin. Nat Commun 6: 7299, 2015.

21. Wang L, Yang H, Palmbos PL, Ney G, Detzler TA, Coleman D, Leflein J, Davis M, Zhang M, Tang W, et al: ATDC/TRIM29 phosphorylation by ATM/MAPKAP kinase 2 mediates radioresistance in pancreatic cancer cells. Cancer Res 74: 1778-1788, 2014.
22. Xu R, Hu J, Zhang T, Jiang C and Wang HY: TRIM29 overexpression is associated with poor prognosis and promotes tumor progression by activating $\mathrm{Wnt} / \beta$-catenin pathway in cervical cancer. Oncotarget 7: 28579-28591, 2016.

23. Dasari S and Tchounwou PB: Cisplatin in cancer therapy: Molecular mechanisms of action. Eur J Pharmacol 740: 364-378, 2014.

24. Yuan Z, Villagra A, Peng L, Coppola D, Glozak M, Sotomayor EM, Chen J, Lane WS and Seto E: The ATDC (TRIM29) protein binds p53 and antagonizes p53-mediated functions. Mol Cell Biol 30: 3004-3015, 2010.

25. Vermeulen K, Van Bockstaele DR and Berneman ZN: The cell cycle: A review of regulation, deregulation and therapeutic targets in cancer. Cell Prolif 36: 131-149, 2003.

26. Malumbres M: Cyclins and related kinases in cancer cells. J BUON 12 (Suppl 1): S45-S52, 2007.

27. Deryugina EI and Quigley JP: Tumor angiogenesis: MMP-mediated induction of intravasation- and metastasissustaining neovasculature. Matrix Biol 44-46: 94-112, 2015.

28. Yoon SO, Park SJ, Yun CH and Chung AS: Roles of matrix metalloproteinases in tumor metastasis and angiogenesis. J Biochem Mol Biol 36: 128-137, 2003.

29. Abarikwu SO and Farombi EO: Atrazine induces apoptosis of SH-SY5Y human neuroblastoma cells via the regulation of Bax/ Bcl-2 ratio and caspase-3-dependent pathway. Pestic Biochem Physiol 118: 90-98, 2015.

30. Hsieh AC, Truitt ML and Ruggero D: Oncogenic AKTivation of translation as a therapeutic target. Br J Cancer 105: 329-336, 2011.

31. Ávalos Y, Canales J, Bravo-Sagua R, Criollo A, Lavandero S and Quest AF: Tumor suppression and promotion by autophagy. BioMed Res Int 2014: 603980, 2014

32. Xing M: Genetic alterations in the phosphatidylinositol-3 kinase/ Akt pathway in thyroid cancer. Thyroid 20: 697-706, 2010.

33. Zhou XM, Sun R, Luo DH, Sun J, Zhang MY, Wang MH, Yang Y, Wang HY and Mai SJ: Upregulated TRIM29 promotes proliferation and metastasis of nasopharyngeal carcinoma via PTEN/AKT/mTOR signal pathway. Oncotarget 7: 1363413650, 2016.

34. Brzezianska E and Pastuszak-Lewandoska D: A minireview: The role of MAPK/ERK and PI3K/Akt pathways in thyroid follicular cell-derived neoplasm. Front Biosci (Landmark Ed) 16: 422-439, 2011. 